\title{
$\alpha$-Lipoic Acid and Superoxide Dismutase in the Management of Chronic Neck Pain: A Prospective Randomized Study
}

\author{
Giulia Letizia Mauro • Pietro Cataldo • \\ Giuseppa Barbera $\cdot$ Antonio Sanfilippo
}

Published online: 30 January 2014

(c) The Author(s) 2014. This article is published with open access at Springerlink.com

\begin{abstract}
Background and Objective Since oxidative stress plays a pathogenetic role in chronic neck pain (CNP), we investigated whether a combination of $\alpha$-lipoic acid (ALA) and superoxide dismutase (SOD) might improve pain control and the efficacy of physiotherapy ("multimodal therapy") in patients with CNP.

Setting This study was conducted in the Rehabilitation Unit of the Department of Surgical and Oncological Sciences at the University Policlinic in Palermo, Italy.

Design and Patients This was a prospective, randomized, open study in outpatients.

Intervention Patients randomly received either physiotherapy alone (group $2 ; n=45$ ) or a combination of ALA $600 \mathrm{mg}$ and SOD $140 \mathrm{IU}$ daily in addition to physiotherapy (group $1 ; n=51$ ), for 60 days. Pain was assessed by a visual analogue scale (VAS) and a modified Neck Pain Questionnaire (mNPQ). Treatment compliance and safety were also evaluated.

Results Both groups experienced a significant reduction in the VAS and mNPQ scores after 1 month; however, while no further improvement was observed in group 2 at 60 days, group 1 showed a further VAS reduction $(p<0.001)$. In addition, in the mNPQ at 60 days, more patients in group 1 than in group 2 reported that their neck pain was improved $(p<0.01)$, and they showed greater
\end{abstract}

G. Letizia Mauro $(\bowtie) \cdot$ P. Cataldo · G. Barbera

Cattedra di Medicina Fisica e Riabilitativa, Università degli Studi di Palermo, Via Del Vespro 127, 90127 Palermo, Italy e-mail: giulia.letiziamauro@unipa.it

\section{A. Sanfilippo}

Clinica Ortopedica e Traumatologica, Dipartimento di Discipline Chirurgiche, Oncologiche e Stomatologiche, Università degli Studi di Palermo, Via Del Vespro 127, 90127 Palermo, Italy compliance with prescribed physiotherapy $(p=0.048)$. No drug reaction was observed.

Conclusion Use of ALA/SOD in combination with physiotherapy may be a useful approach to CNP, being antioxidants that act on nerve inflammation and disease progression.

Clinical Rehabilitation Impact These preliminary observations suggest that some interesting goals (better pain control and physical wellbeing) can be achieved by multimodal therapy in CNP patients.

\section{Introduction}

Cervical spinal pain is defined as a pain perceived anywhere in the posterior region of the cervical spine, from the superior nuchal line to the first thoracic spinous process [1] or, alternatively, as a pain located in the anatomical region of the neck, either with or without radiation to the head, trunk, and upper limbs [2]. The history of cervical spinal pain usually includes an acute phase (which is sustained by mechanical stimulation of cervical intervertebral discs, cervical facet joints, atlanto-axial and atlanto-occipital joints, ligaments, fascia, muscles, and nerve root dura, which are capable of transmitting pain in the cervical spine with resulting symptoms of neck pain, upper extremity pain, and headache) and a chronic phase (which is sustained by inflammation and myelin axonal degeneration, with the characteristics of neuropathic pain).

Chronic neck pain (CNP) is often described as widespread hyperalgesia of the skin, ligaments, and muscles on palpation and on both passive and active movements in the neck and shoulder area [3]. CNP affects between 50 and $75 \%$ of people who experience acute neck pain initially [4-6], and it is estimated to have an annual prevalence 
between 30 and $50 \%$ [7, 8], being associated with significant economic, societal, and health effects [5, 8-10]. The effective treatment of CNP is still an outstanding issue; guidelines on pain agree on considering multimodal therapy (i.e. a combination of active principles with complementary mechanisms) as the best strategy to improve efficacy and tolerability [11-13].

Increased oxidative stress plays a pivotal role in neuropathic pain, leading to axonal degeneration and myelin degradation. Reactive oxygen species (ROS) promote nerve inflammation through enhanced synthesis of inflammatory cytokines and chemotactic molecules, which recall and activate leukocytes. In such a way, the ROStriggered inflammatory process leads to pain and loss of nerve conduction functionality, and use of antioxidants could represent a suitable strategy for CNP $[14,15]$.

Among antioxidant agents, the free radical scavenger $\alpha$ lipoic acid (ALA) has recently been proposed for use in patients with peripheral nerve injuries and other neuropathies [16]. ALA has documented efficacy in treatment of diabetic neuropathy [17], where it reduces pain and symptoms of peripheral neuropathy $[18,19]$ and improves nerve conduction [13, 20]. Recent studies have shown that ALA also reduces pain, paresthesia, and numbness in patients with compressive radiculopathy syndrome from disc-nerve root conflict [21] and other types of neuropathies, such as carpal tunnel syndrome [22]. In addition, combination treatment with ALA and $\gamma$-linolenic acid within a rehabilitation program for 6 weeks reduced sensory symptoms and neuropathic pain in patients with compressive radiculopathy syndrome from disc-nerve root conflict, compared with patients undergoing a rehabilitation program alone for 6 weeks [23, 24].

Superoxide dismutase (SOD) is one of the most important antioxidant enzymes, being responsible for neutralization of superoxide, the free radical occurring in the cellular respiration. SOD is endowed with a powerful anti-inflammatory action due to its antioxidant property and direct action on neutrophils, inducing their apoptosis; thus, SOD has a key role in inhibiting the inflammatory response, which is closely correlated with attenuation of hyperalgesia [25]. Furthermore, SOD inhibits biosynthesis of some principal inflammatory cytokines and avoids apoptosis of nerves [26]. Since during inflammationwhether acute or chronic-endogenous SOD is not sufficient to completely neutralize oxygen free radicals, dietary supplementation of SOD has been investigated in some diseases, such as arthritis [27], and it has been shown that orally administered SOD not only has antioxidant activity but also works as an effective nerve protector [28, 29].

With this background in mind, our attention was captured by a marketed combination of ALA $600 \mathrm{mg}$ and SOD $140 \mathrm{IU}$ and, therefore, we aimed to investigate its efficacy on sensory symptoms and neuropathic pain in patients with $\mathrm{CNP}$, when added to a standard rehabilitation program (physiotherapy), compared with the rehabilitation program alone. We hypothesized that the proposed multimodal approach would improve most of the clinical parameters and that it would be more effective than physiotherapy alone.

\section{Patients and Methods}

In accordance with a prospective, randomized, open study design, patients were screened between March 2010 and April 2011 in the Rehabilitation Unit of the Department of Surgical and Oncological Sciences at the University Policlinic in Palermo, Italy. All participants were recruited from consecutive new patients presenting to an interventional pain management practice with CNP.

Patients with a history of chronic function-limiting neck pain lasting at least 3 months were included in the study. CNP was defined as pain perceived anywhere in the posterior region of the cervical spine, from the superior nuchal line to the first thoracic spinous process, often combined with widespread hyperalgesia of the skin, ligaments, and muscles on palpation and both passive and active movements of the neck and shoulder [1]. Other criteria for patient inclusion were age over 18 years, no physiotherapy, no ongoing chiropractic care or rehabilitation for the neck area, ability to provide voluntary written informed consent, willingness to participate in the study as well as follow-up, and ability to perform painful movements of the neck and shoulder.

The exclusion criteria included neck pain due to a motor vehicle accident, neck surgery, severe osteoarthritis or inflammatory arthritis, symptomatic spinal stenosis, surgical interventions of the cervical spine within the previous 3 months, uncontrolled major depression or psychiatric disorder, acute or uncontrolled medical illness (malignancy or active infection), chronic severe condition that could interfere with interpretation of the outcome assessments, pregnancy or lactation, and engagement in experimental medical treatment. Participants with concurrent headaches, non-radicular pain in the upper extremities, and lower back pain were not excluded if neck pain was their main symptom.

The study was approved by the local independent ethics committee, and all patients were informed of the investigational nature of the study. After the patients had read the study information and signed the informed consent form, they were physically examined. The height and weight were measured, and the body mass index (BMI) was calculated. Gender, age, and occupation were documented, as well as other clinical characteristics such as the diagnosis, 
time since first diagnosis, medical history, diagnostic tests performed, duration of therapy, and concomitant treatments.

According to a computer-generated random allocation sequence, patients were randomly assigned either to a group treated with a combination of ALA $600 \mathrm{mg}$ and SOD 140 IU once daily in addition to physiotherapy (group 1), or to a group receiving physiotherapy alone (group 2). The ALA/SOD combination therapy was purchased by the patients from a pharmacy. Both groups were treated and followed up for two consecutive months. Patients were not allowed to take any other analgesic compound for the entire duration of the study.

Cervicobrachial pain was assessed by the patients by means of a visual analogue scale (VAS) and a modified Neck Pain Questionnaire (mNPQ). Both the VAS and the mNPQ questionnaire were administered at baseline (T0, pre-treatment), and after 1 month (T1) and 2 months (T2) of treatment.

The VAS is a $100 \mathrm{~mm}$ line, oriented vertically or horizontally, with one end representing "no pain" and the other end representing "pain as bad as it can be". The patient is asked to mark a place on the line corresponding to their current pain intensity. The VAS is the most frequently used pain measure because it is simple to use and has good psychometric properties [30]. The VAS assessment was done in a resting position ("pain at rest") and during a pain-provoking movement, such as neck flexion, neck extension, lateral neck flexion, and neck rotation in either direction ("pain on movement").

The mNPQ was developed to measure neck pain and consequent patient disability and wellbeing. It is relatively simple to use and provides an objective measure for monitoring symptoms over time, according to ten questions about (1) neck pain intensity; (2) neck pain and sleeping; (3) pins and needles or numbness in the arms at night; (4) duration of symptoms; (5) carrying; (6) reading and watching television; (7) working and/or housework; (8) social activities; (9) driving; and (10) comparison between the current state and the last time the questionnaire was completed. Each question has a 5-point scaled answer, from 0 (no pain or no interference with life/activities) to 5 (severe pain or inability to perform activities). Question \#9 about driving was omitted if the patient did not drive a car when in good health, and question \#10 was given only at the control visits ( $\mathrm{T} 1$ and $\mathrm{T} 2$ ), compared with the previous visits [baseline (T0) and T1, respectively]. The "neck pain score" was calculated as the sum of the points for the first nine questions. If all nine questions were answered, then NPQ percentage $=($ neck pain score $) / 36 \times 100 \%$. If only the first eight questions were answered, then NPQ percentage $=($ neck pain score $) / 32 \times 100 \%$. The answer to question \#10 was analyzed separately. The percentages ranged from 0 to $100 \%$. The higher the percentage, the greater the disability $[31,32]$.

The compliance of the patients with the study was assessed by checking whether the patients followed the physiotherapy sessions that were prescribed at the start of the study and, only in group 1, whether the patients had missed some therapies because of adverse reactions, intolerance, or "lack of efficacy" as perceived by the patients. In the case of adverse event or drug reactions, the patients were asked to report which reaction occurred, how long it lasted, and which measures were undertaken to control the reaction (treatment stopped, concomitant therapies, etc.).

The primary study objective was improvement of pain. The primary outcomes were changes in the VAS and mNPQ scores; the secondary objectives were compliance with medical prescriptions (which was also considered to be an indirect assessment of efficacy) and safety.

The results are reported as descriptive statistics: quantitative parameters are reported as means, minimums, maximums and standard deviations; qualitative parameters are reported as absolute and relative frequencies. Comparisons were made with a chi-squared test for qualitative parameters and with a paired Student's $t$ test for quantitative ones. Analysis of variance (ANOVA) and analysis of covariance (ANCOVA) of the VAS at the baseline visit were performed to test variations in parameters through time and between groups. $P$ values were considered statistically significant if $<0.05$ (confidence interval $95 \%$ ). Statistical analyses were performed with SPSS Statistical Package, version 13.0 (SPSS Italia Srl/Inc.).

\section{Results}

Ninety-eight patients were screened and randomized into the study. Two patients were excluded from the data cleaning because their control visits ( $\mathrm{T} 1$ and $\mathrm{T} 2$ ) were missing and, therefore, no efficacy data were available. The final database consisted of 96 patients (11 males and 85 females) with a mean age of $53.2 \pm 14.1$ years (range 20-83). All patients had a diagnosis of chronic cervicobrachial pain; of those, 51 patients were treated with the combination of ALA/SOD in addition to physiotherapy, while the other 45 patients had physiotherapy alone (Table 1).

The most frequently prescribed types of physiotherapy in the medical history were diadynamic, carbon dioxide laser, ionophoresis, transcutaneous electrical nerve stimulation (TENS), massage therapy, and functional rehabilitation. Details are reported in Table 2.

Both ALA/SOD combined with physiotherapy and physiotherapy alone achieved a significant reduction in 
Table 1 Demographic and clinical characteristics of the patients

\begin{tabular}{llll}
\hline & Total, $n=96$ & ALA/SOD + physiotherapy, $n=51$ & Physiotherapy alone, $n=45$ \\
\hline Males/females & $11 / 85$ & $6 / 45$ & $5 / 40$ \\
Age [years] & $53.2 \pm 14.1(20-83)$ & $52.7 \pm 13.7(20-81)$ & $53.8 \pm 14.6(20-83)$ \\
Weight $[\mathrm{kg}]$ & $67.3 \pm 12.1(47-100)$ & $69.3 \pm 13.4(47-100)$ & $65.1 \pm 10.2(48-95)$ \\
Height [cm] & $161.3 \pm 7.4(147-180)$ & $160.9 \pm 7.5(148-180)$ & $161.7 \pm 7.3(147-180)$ \\
BMI [kg/m $\left.{ }^{2}\right]$ & $25.8 \pm 4.4(16.1-35.2)$ & $26.6 \pm 4.7(16.1-35.2)$ & $24.9 \pm 3.9(16.9-34.2)$ \\
Occupation & & & $19(42.2 \%)$ \\
Housewife & $45(46.9 \%)$ & $26(51.0 \%)$ & $3(6.7 \%)$ \\
Pensioner & $10(10.4 \%)$ & $7(13.7 \%)$ & $4(8.9 \%)$ \\
Employer & $5(5.2 \%)$ & $1(2.0 \%$ & $19(42.2 \%)$ \\
Other & $36(37.5 \%)$ & $17(33.3 \%)$ & $42(93.3 \%)$ \\
Diagnosis & & $51(100 \%)$ & $18(42.9 \%)$ \\
Cervicobrachial pain & $93(96.9 \%)$ & $28(54.9 \%)$ & $13(31.0 \%)$ \\
Bilateral & $46(49.5 \%)$ & $3(5.9 \%)$ & $6(14.3 \%)$ \\
Right side & $16(17.2 \%)$ & $7(13.7 \%)$ & $5(11.8 \%)$ \\
Left side & $13(14.0 \%)$ & $13(25.5 \%)$ & 1 \\
Unknown & $18(19.3 \%)$ & - & 1 \\
Cervical arthrosis & 1 & - & 1 \\
Cervical muscle tension & 1 & 1 &
\end{tabular}

The results are reported as means \pm standard deviations with minimum-maximum ranges in parentheses, or as absolute and relative frequencies, as appropriate. No statistically significant difference was observed between the groups

$A L A \alpha$-lipoic acid, BMI body mass index, SOD superoxide dismutase

Table 2 Types of physiotherapy prescribed for patients enrolled in the study

$A L A$-lipoic acid, $S O D$ superoxide TENS

transcutaneous electrical nerve stimulation

\begin{tabular}{llll}
\hline Type of therapy & All patients, $n=96$ & $\begin{array}{l}\text { ALA/SOD plus } \\
\text { physiotherapy, } n=51\end{array}$ & $\begin{array}{l}\text { Physiotherapy } \\
\text { alone, } n=45\end{array}$ \\
\hline Diadynamic & $61(64 \%)$ & $34(67 \%)$ & $27(60 \%)$ \\
Carbon dioxide laser & $58(60 \%)$ & $33(65 \%)$ & $25(56 \%)$ \\
Radar & $45(47 \%)$ & $19(37 \%)$ & $26(58 \%)$ \\
Ionophoresis & $37(39 \%)$ & $16(31 \%)$ & $21(47 \%)$ \\
TENS & $21(22 \%)$ & $12(24 \%)$ & $9(20 \%)$ \\
Massage & $11(11 \%)$ & $6(12 \%)$ & $5(11 \%)$ \\
Functional rehabilitation & $11(11 \%)$ & $4(8 \%)$ & $7(16 \%)$ \\
Ultrasound & $3(3 \%)$ & $1(2 \%)$ & $2(4 \%)$ \\
Electroanalgesia & $1(1 \%)$ & - & $1(2 \%)$ \\
Infrared & $1(1 \%)$ & - & $1(2 \%)$ \\
Magnetic & $1(1 \%)$ & - & $1(2 \%)$ \\
Rehabilitation & $1(1 \%)$ & - & $1(2 \%)$ \\
\hline
\end{tabular}

"pain at rest" perceived by the patients, as was demonstrated by the VAS score, which decreased in group 1 from $60.8 \pm 21.5$ (at T0) to $42.4 \pm 22.1$ (at T1) and $27.4 \pm 22.5$ (at the end of treatment, T2), and in group 2 from $61.3 \pm 20.5$ (at T0) to $42.0 \pm 23.6$ (at T1) and $39.2 \pm 20.1$ (at T2). It is noteworthy to mention that after 60 days of treatment, the "pain at rest" was significantly lesser in patients receiving ALA/SOD in addition to physiotherapy than in those treated with physiotherapy alone $(p<0.005)$ (Table 3$)$.

Also, the VAS for "pain on movement" induced by movements of the neck and/or shoulder performed by the physicians was significantly reduced in group 1 from $70.4 \pm 19.7$ (at T0) to $47.5 \pm 21.2$ (at T1) and $31.8 \pm 20.8$ (at T2); and in group 2 it was reduced from $73.0 \pm 19.5$ (at T0) to $47.2 \pm 24.8$ (at T1) and 
Table 3 Visual analogue scale (VAS) scores assessing "pain at rest" and "pain on movement" in patients treated with $\alpha$-lipoic acid (ALA) and superoxide dismutase (SOD) plus physiotherapy, versus physiotherapy alone

\begin{tabular}{|c|c|c|}
\hline & ALA/SOD plus physiotherapy & Physiotherapy alone \\
\hline \multicolumn{3}{|c|}{ VAS "pain at rest" } \\
\hline Baseline & $60.8 \pm 21.5$ & $61.3 \pm 20.5$ \\
\hline 30 days & $42.4 \pm 22.1$ & $42.0 \pm 23.6$ \\
\hline 60 days & $27.4 \pm 22.5^{* * *},,^{\circ \circ}$ & $39.2 \pm 20.1 * * *$ \\
\hline \multicolumn{3}{|c|}{ VAS "pain on movement" } \\
\hline Baseline & $70.4 \pm 19.7$ & $73.0 \pm 19.5$ \\
\hline 30 days & $47.5 \pm 21.2$ & $47.2 \pm 24.8$ \\
\hline 60 days & $31.8 \pm 20.8^{* * *},{ }^{\circ \circ}$ & $44.2 \pm 22.4 * * *$ \\
\hline
\end{tabular}

The results are reported as means \pm standard deviations

Statistically significant differences on ANOVA within groups: $* * * p<0.001$ versus baseline; statistically significant differences on ANCOVA between groups: ${ }^{\circ 0} p<0.01$ and ${ }^{\circ 00} p<0.005$ versus physiotherapy alone

ANCOVA analysis of covariance, ANOVA analysis of variance

$44.2 \pm 22.4$ (at T2). Again, the ANCOVA (for the VAS covariate at the baseline visit) between the two groups after 60 days of treatment showed a statistically significant difference in favor of the group treated with ALA/SOD in addition to physiotherapy, versus physiotherapy alone $(p<0.01)$ (Table 3).

The reduced VAS score was reflected by the reduction in $\mathrm{mNPQ}$ scores. The average $\mathrm{mNPQ}$ percentage decreased from $41.7 \pm 16.6$ at baseline to $24.4 \pm 14.8$ after 30 days and $17.6 \pm 13.9$ after 60 days of treatment in group 1 $(p<0.001)$, and from $44.4 \pm 15.8$ at baseline to $23.1 \pm 13.9$ after 1 month and $17.0 \pm 10.4$ after 2 months in group $2(p<0.001)$. There was no statistically significant difference between the groups. However, the last question of the mNPQ questionnaire ("In comparison with the last time you answered the questionnaire, neck pain is...") confirmed the results achieved on the VAS scale. After 2 months of treatment, more than $81 \%$ of patients receiving ALA/SOD in addition to physiotherapy were improved, either "much improved" or "slightly improved", compared with only $29 \%$ of patients treated with physiotherapy alone. The difference between the groups was statistically significant $(p<0.001)$ (Fig. 1).

Lastly, compliance with the treatment was checked by the physician. In group 1 receiving ALA/SOD in addition to physiotherapy, more than 84 and $78 \%$ of patients were reported to have followed the medical prescriptions for physiotherapy after 30 and 60 days of treatment, respectively. Conversely, at the same time points, only 71 and $55 \%$ of patients in group 2 were reported to be compliant with the prescriptions for physiotherapy, and most of them reported that they were not completely happy about the

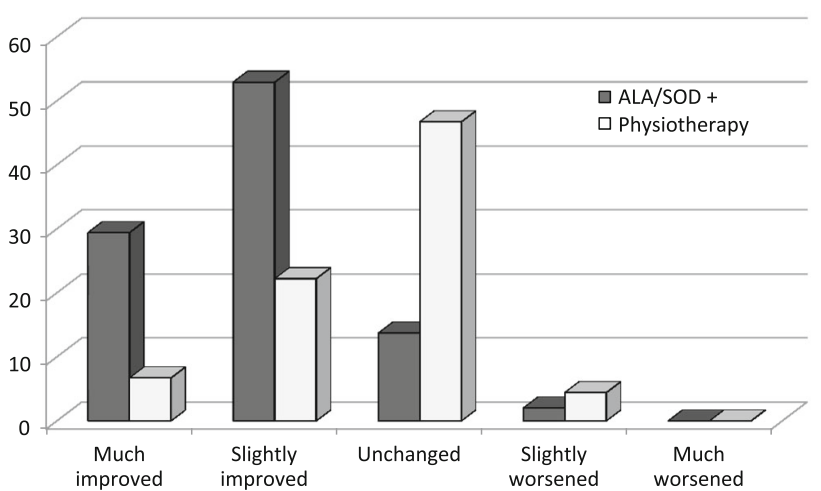

Fig. 1 Scores for modified Neck Pain Questionnaire (mNPQ) question \#10: "Compared with the last time you completed the questionnaire, is the neck pain...". After 2 months of treatment (at $\mathrm{T} 2$ ), the difference between groups was statistically significant, according to a chi-squared test $(p<0.001)$. ALA $\alpha$-lipoic acid, SOD superoxide dismutase

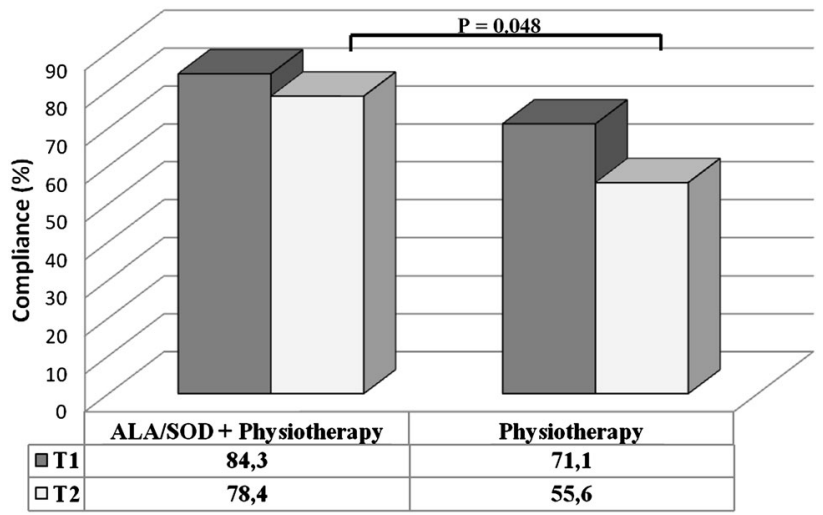

Fig. 2 Percentages of patients who fully complied with physiotherapy prescribed by the site medical staff, in the group treated with $\alpha$ lipoic acid (ALA) and superoxide dismutase (SOD) plus physiotherapy, and in the group treated with physiotherapy alone. The difference between groups was statistically significant $(p=0.048)$

results achieved with physiotherapy alone. The difference between the groups was significant $(p=0.048)$ and was considered an indirect confirmation that better pain control was achieved in group 1 than in group 2 (Fig. 2).

The tolerability was generally acceptable in both experimental groups, and no drug-related adverse events were reported.

\section{Discussion}

Cervicobrachial pain is a common cervical spine disorder. When the condition evolves to chronicity (CNP), it encompasses the characteristics of neuropathic pain and becomes a persistent or recurring problem, which impacts unfavorably on an individual's mental as well as physical 
health, thus leading to high costs for the health care system and society [33].

Here, we report the results of a prospective, randomized, controlled study aimed at evaluating the difference in pain relief between physical rehabilitation alone and multimodal therapy in patients affected by CNP. Our results demonstrated a statistically significant difference between the two study groups, confirming the hypothesis that multimodal therapy, combining oral antioxidants-ALA and SODwith physiotherapy, would lead to better improvement of perceived pain in these patients. In addition, both groups reported improvements after the first month of treatment, but after 2 months, group 2 (who were treated with physiotherapy alone) stopped improving, while patients in group 1 receiving $\mathrm{ALA}_{600} \mathrm{SOD}^{\circledR}$ continued to experience improvement in their perceived pain, as showed by their mNPQ responses.

ALA is a biological compound occurring in foods such as liver, spinach, and broccoli, but it is always covalently bound to macromolecules and, in fact, it is not fully bioavailable from standard dietary sources. Additionally, the amount of ALA that is present in the diet is very small, and dietary supplementation is needed whenever increased oxidative stress in the body (e.g. because of chronic inflammation or other stressful conditions) leads to clinically significant consumption of endogenous antioxidants.

$\mathrm{ALA}_{600} \mathrm{SOD}^{\circledR}$ is an oral formulation and is characterized by rapid absorption, high bioavailability, a short halflife, and low toxicity [34]. These findings could significantly improve the clinical benefit and therapeutic effects of lipoic acid at the cellular level, thus making $\mathrm{ALA}_{600-}$ $\mathrm{SOD}^{\circledR}$ a suitable formulation for long-term administration in chronic conditions, such as peripheral neuropathies.

Treatment with $\mathrm{ALA}_{600} \mathrm{SOD}^{\circledR}$ for 4 months led patients with diabetic neuropathy to experience a significant improvement in their electroneurographic parameters and perception of pain. The best improvements were observed in sensory nerve conduction, thus confirming that a combination of two powerful antioxidant agents leads to improvement in both subjective and objective parameters in patients with diabetic neuropathy [35].

The results of our study suggest that important goals can be achieved in the treatment of cervicobrachial pain by combining physiotherapy with oral antioxidants, i.e. optimized pain control, enhanced functional abilities and physical and psychological wellbeing, enhanced quality of life, and minimized adverse effects. Thus, $\mathrm{ALA}_{600} \mathrm{SOD}^{\circledR}$ may represent a powerful adjuvant in the treatment of cervicobrachial pain.

The limitations of our study may be represented by the small sample size, which reduced the possibility of extrapolating the results to other patient populations. The study was not blinded, and long term outcomes were not assessed; successfully treated patients should be followed up to determine whether the outcome was sustained. The measures that were reported were self-report tools. Although self-report tools might be considered the most directly reliable means of obtaining such information, potential issues with the credibility of responses should be acknowledged.

In the absence of comparable data in the literature, this study must be considered a pilot one; however, reliability of the study results is suggested by other considerations. Among the concomitant therapies taken by patients, there were no analgesics, thus no bias in assessing the reduction of perceived pain occurred. Since the definition of cervicobrachial pain is often ambiguous, the diagnosis was made for all enrolled patients at the same hospital by the same medical staff, avoiding bias in the definition of the disease. No adverse events were recorded during the study, confirming that few or no side effects were induced by $\mathrm{ALA}_{600} \mathrm{SOD}^{\circledR}$.

Although CNP and neuropathic pain still remain difficult to manage, the results of our study suggest that the combination of ALA/SOD and physiotherapy may be a useful approach in the management of these patients.

\section{Conclusion}

Multidisciplinary interventions represent multimodality approaches in the context of a treatment program that includes more than one discipline. Our results and the literature indicate that use of multidisciplinary treatment programs is effective in reducing the intensity of pain reported by patients. Supplementation with a combination of antioxidant agents represents an etiopathogenetic approach unlike that of analgesics, which is purely symptomatic. This multimodal approach can reduce symptomatology and avoid progression of disease, while also promoting direct anti-inflammatory effects on nerves.

Acknowledgments No funding source had any role in the study design; collection, analysis, or interpretation of the data; or in the preparation of this report.

Conflict of interest The authors declare that they have no conflicts of interest to disclose.

Open Access This article is distributed under the terms of the Creative Commons Attribution Noncommercial License which permits any noncommercial use, distribution, and reproduction in any medium, provided the original author(s) and the source are credited.

\section{References}

1. Merskey H, Bogduk N. Classification of chronic pain: descriptions of chronic pain syndromes and definitions of pain terms. 2nd ed. Seattle: IASP Press; 1994. 
2. Guzmán J, Hurwitz EL, Carroll LJ, Haldeman S, Cote P, Carragee EJ. A new conceptual model of neck pain. Linking onset, course, and care: the Bone and Joint Decade 2000-2010 Task Force on Neck Pain and Its Associated Disorders. Spine. 2008;33:S14-23.

3. Ylinen J. Physical exercises and functional rehabilitation for the management of chronic neck pain. Eura Medicophys. 2007;43:119-32.

4. Côté P, Cassidy JD, Carroll LJ, Kristman V. The annual incidence and course of neck pain in the general population: a population-based cohort study. Pain. 2004;112:267-73.

5. Martin BI, Deyo RA, Mirza SK, Turner JA, Comstock BA, Hollingworth W, Sullivan SD. Expenditures and health status among adults with back and neck problems. JAMA. 2008;299:656-64.

6. Thelin A, Holmberg S, Thelin N. Functioning in neck and low back pain from a 12-year perspective: a prospective populationbased study. J Rehabil Med. 2008;40:555-61.

7. Hogg-Johnson S, van der Velde G, Carroll LJ, Holm LW, Cassidy JD, Guzman J, Côté P, Haldeman S, Ammendolia C, Carragee E, Hurwitz E, Nordin M, Peloso P, Bone and Joint Decade 2000-2010 Task Force on Neck Pain and Its Associated Disorders. The burden and determinants of neck pain in the general population: results of the Bone and Joint Decade 2000-2010 Task Force on Neck Pain and Its Associated Disorders. Spine. 2008;33:S39-51.

8. Manchikanti L, Singh V, Datta S, Cohen SP, Hirsch JA. Comprehensive review of epidemiology, scope, and impact of spinal pain. Pain Physician. 2009;12:E35-70.

9. Côté P, van der Velde G, Cassidy JD, Carroll LJ, Hogg-Johnson S, Holm LW, Carragee EJ, Haldeman S, Nordin M, Hurwitz EL, Guzman J, Peloso PM, Bone and Joint Decade 2000-2010 Task Force on Neck Pain and Its Associated Disorders. The burden and determinants of neck pain in workers. Results of the Bone and Joint Decade 2000-2010 Task Force on Neck Pain and Its Associated Disorders. Spine. 2008;33:S60-74.

10. Manchikanti L, Pampati V, Boswell MV, Smith HS, Hirsch JA. Analysis of the growth of epidural injections and costs in the Medicare population: a comparative evaluation of 1997, 2002, and 2006 data. Pain Physician. 2010;13:199-212.

11. Guzmán J, Esmail R, Karjalainen K, Malmivaara A, Irvin E, Bombardier C. Withdrawn: multidisciplinary bio-psycho-social rehabilitation for chronic low-back pain. Cochrane Database Syst Rev. 2007;(2):CD000963.

12. Karjalainen K, Malmivaara A, Van Tulder M, Roine R, Jauhiainen M, Hurri H. Multidisciplinary biopsychosocial rehabilitation for neck and shoulder pain among working age adults (Cochrane review). The Cochrane Library. Chichester: John Wiley and Sons, Ltd.; 2006.

13. Lee FH, Raja SN. Complementary and alternative medicine in chronic pain. Pain. 2011;152:28-30.

14. Viggiano E, Monda M, Viggiano A, Viggiano A, Aurilio C, De Luca B. Persistent facial pain increases superoxide anion production in the spinal trigeminal nucleus. Mol Cell Biochem. 2010;339:149-54.

15. Schwartz ES, Kim HY, Wang J, Lee I, Klann E, Chung JM, et al. Persistent pain is dependent on spinal mitochondrial antioxidant levels. J Neurosci. 2009;29:159-68.

16. Ranieri M, Sciuscio M, Cortese AM, Stasi M, Panza F, Megna M, et al. Possible role of alpha-lipoic acid in the treatment of peripheral nerve injuries. J Brachial Plex Peripher Nerve Injury. 2010;5:15-20.

17. Ziegler D. Thioctic acid for patients with symptomatic diabetic polyneuropathy: a critical review. Treat Endocrinol. 2004;3:173-89.
18. Bilska A, Wlodek L. Lipoic acid: the drug of the future? Pharm Rep. 2005;57:570-7.

19. Mitsui Y, Schmelzer JD, Zollman PJ, Mitsui M, Trischeler HJ, Low PA. Lipoic acid provides neuroprotection from ischemiareperfusion injury of peripheral nerve. $J$ Neurol Sci. 1999;163:11-6.

20. Androne L, Gavan NA, Veresiu IA, Orasan R. In vivo effect of lipoic acid on lipid peroxidation in patients with diabetic neuropathy. In Vivo. 2000;14:327-30.

21. Memeo A, Loiero M. Thioctic acid and acetyl-L-carnitine in the treatment of sciatic pain caused by herniated disc. Clin Drug Investig. 2008;28:495-500.

22. Di Geronimo G, Fonzone Caccese A, Caruso L, Soldati A, Passaretti U. Treatment of carpal tunnel syndrome with $\alpha$-lipoic acid. Eur Rev Med Pharmacol Sci. 2009;13:133-9.

23. Ranieri M, Sciuscio M, Musci L, Ciullo F, Cortese AM, Chiumarulo P, Putignano P, Santamato A, Ineri G, Megna M, Megna G, Stasi M. Efficacia e sicurezza della supplementazione con acido alfa-lipoico (ALA) e acido gamma-linolenico (GLA) nel trattamento della rachialgia: studio osservazionale preliminare. Eur Med Phys. 2008;44(Suppl):1-4.

24. Ranieri M, Sciuscio M, Cortese AM, Santamato A, Di Teo L, Ianieri G, Bellomo RG, Stasi M, Megna M. The use of alphalipoic acid (ALA), gamma linolenic acid (GLA) and rehabilitation in the treatment of back pain: effect on health-related quality of life. Int J Immunopathol Pharmacol. 2009;22(3 Suppl):45-50.

25. Wang ZQ, Porreca F, Cuzzocrea S, et al. A newly identified role for superoxide in inflammatory pain. J Pharmacol Exp Ther. 2004;309:869-78.

26. Yasui K, Baba A. Therapeutic potential of superoxide dismutase (SOD) for resolution of inflammation. Inflamm Res. 2006; 55:359-63.

27. Cuzzocrea S, Riley DP, Caputi AP, Salvemini D. Antioxidant therapy: a new pharmacological approach in shock, inflammation and ischemia/reperfusion injury. Pharmacol Rev. 2001;53:135-59.

28. Milesi MA, Lacan D, Brosse H, Didier D, Notin C. Effect of an oral supplementation with a proprietary melon juice concentrate (Extramel) on stress and fatigue in healthy people: a pilot, double-blind, placebo-controlled clinical trial. Nutr J. 2009;8:40.

29. Nakajima S, Ohsawa I, Nagata K, Ohta S, Ohno M, Ijichi T, Mikami T. Oral supplementation with melon superoxide dismutase extract promotes antioxidant defences in the brain and prevents stress-induced impairment of spatial memory. Behav Brain Res. 2009;200:15-21.

30. Price DD, McGrath PA, Rafii A, Buckingham B. The validation of visual analogue scales as ratio scale measures for chronic and experimental pain. Pain. 1983;17:45-56.

31. Leak AM, Cooper J, Dver S, Williams KA, Turner-Stokes L, Frank AO. The Northwick Park Neck Pain Questionnaire devised to measure neck pain and disability. $\mathrm{Br} \mathrm{J}$ Rheumatol. 1994;33:469-74.

32. Raffaetà G, Mengoni A, Togo R. Studio sperimentale: applicazione terapeutica della tecarterapia nelle sindromi algiche cervicali. Eur Med Phys. 2007;43(Suppl 1):12-8.

33. Daffner S, Hilibrand A, Hanscom B, Brislin B, Vaccaro A, Albert T. Impact on neck and arm pain on overall health status. Spine. 2003;28:817-24.

34. Bertolotto F, Massone A. Combination of alpha lipoic acid and superoxide dismutase leads to physiological and symptomatic improvements in diabetic neuropathy. Drugs RD. 2012;12:1-6.

35. Mignini F, Capacchietti M, Napolioni V, Reggiardo G, Fasani R, Ferrari P. Single dose bioavailability and pharmacokinetic study of a innovative formulation of $\alpha$-lipoic acid $\left(\mathrm{ALA}_{600}\right)$ in healthy volunteers. Miner Med. 2011;102:1-8. 